\title{
Organisational interventions for promoting person-centred care for people with dementia (Protocol)
}

Chenoweth L, Stein-Parbury J, Lapkin S, Wang YA
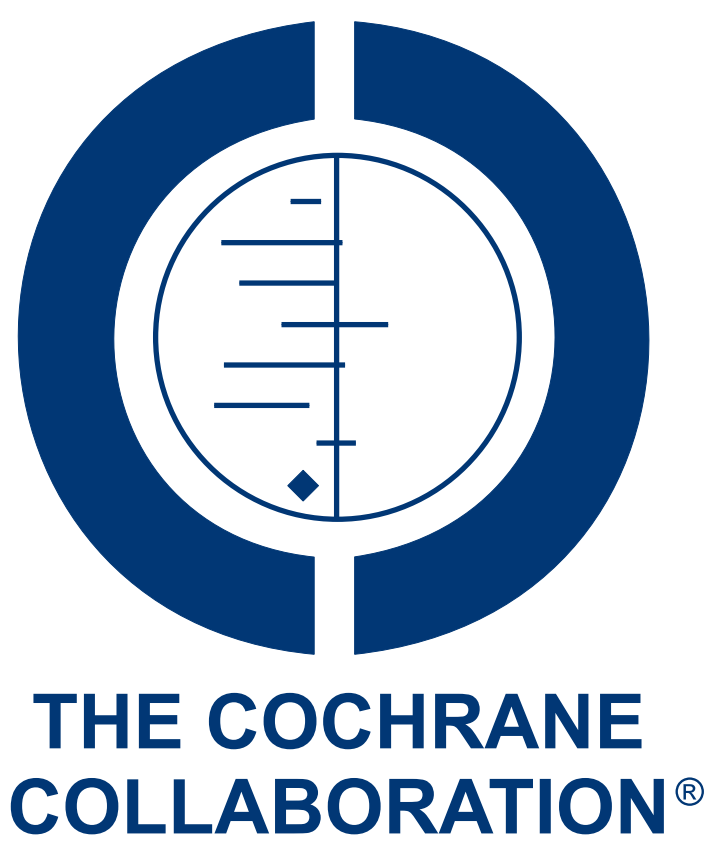

This is a reprint of a Cochrane protocol, prepared and maintained by The Cochrane Collaboration and published in The Cochrane Library 2015, Issue 11

http://www.thecochranelibrary.com

\section{WILEY}

Organisational interventions for promoting person-centred care for people with dementia (Protocol)

Copyright $\odot 2015$ The Cochrane Collaboration. Published by John Wiley \& Sons, Ltd. 
TABLE OF CONTENTS

HEADER . . . . . . . . . . . . . . . . . . . . . . . . . . . . . . . . . . . 1

ABSTRACT . . . . . . . . . . . . . . . . . . . . . . . . . . . . . . . . . . . . . . . 1

BACKGROUND . . . . . . . . . . . . . . . . . . . . . . . . . . . . . . . . . . . .

OBJECTIVES . . . . . . . . . . . . . . . . . . . . . . . . . . . . . . . . . . . . . . . . . .

METHODS . . . . . . . . . . . . . . . . . . . . . . . . . . . . . . . . . . . . . . 44

REFERENCES . . . . . . . . . . . . . . . . . . . . . . . . . . . . . . . . . . . . . . .

APPENDICES . . . . . . . . . . . . . . . . . . . . . . . . . . . . . . . . . . . . . . . . 99

CONTRIBUTIONS OF AUTHORS . . . . . . . . . . . . . . . . . . . . . . . . . . . . . . . . . . . . . . 10

DECLARATIONS OF INTEREST . . . . . . . . . . . . . . . . . . . . . . . . . . . . . . . . 11

SOURCES OF SUPPORT . . . . . . . . . . . . . . . . . . . . . . . . . . . . . . . . . . 11

Organisational interventions for promoting person-centred care for people with dementia (Protocol)

Copyright $\odot 2015$ The Cochrane Collaboration. Published by John Wiley \& Sons, Ltd. 


\title{
[Intervention Protocol]
}

\section{Organisational interventions for promoting person-centred care for people with dementia}

\author{
Lynn Chenoweth ${ }^{1,2}$, Jane Stein-Parbury ${ }^{1}$, Samuel Lapkin ${ }^{1}$, Yueping Alex Wang ${ }^{1}$ \\ ${ }^{1}$ Faculty of Health, University of Technology Sydney, Ultimo, Australia. ${ }^{2}$ Centre for Healthy Brain Ageing, University of New South \\ Wales, Randwick, Australia
}

Contact address: Lynn Chenoweth, Faculty of Health, University of Technology Sydney, Ultimo, New South Wales, 2070, Australia. Lynnette.Chenoweth@uts.edu.au.1.chenoweth@unsw.edu.au.

Editorial group: Cochrane Dementia and Cognitive Improvement Group.

Publication status and date: New, published in Issue 11, 2015.

Citation: Chenoweth L, Stein-Parbury J, Lapkin S, Wang YA. Organisational interventions for promoting person-centred care for people with dementia. Cochrane Database of Systematic Reviews 2015, Issue 11. Art. No.: CD011963. DOI: 10.1002/14651858.CD011963.

Copyright (C) 2015 The Cochrane Collaboration. Published by John Wiley \& Sons, Ltd.

\begin{abstract}
A B S T R A C T
This is the protocol for a review and there is no abstract. The objectives are as follows:

Primary objective: To determine the effectiveness of organisation-wide interventions to implement person-centred care for people with dementia, in relation to reduction of behavioural symptoms such as agitation and depression, improvement in quality of life and functional capabilities, alterations in the use of restraint (physical and/or chemical) and reduction in adverse events.

Secondary objective: To identify the variety, quality, and feasibility of person-centred care approaches for people with dementia, with specific reference to organisational factors which promote and constrain the implementation of person-centred care.
\end{abstract}

\section{B A C K G R O U N D}

\section{Description of the condition}

In developed countries, dementia is one of the leading causes of disability in people aged over 65 years. It occurs in people of all cultures and is becoming increasingly common in countries whose populations are ageing. Worldwide there are over 7.7 million new cases of dementia each year. Approximately 60\% of this disease burden and the most rapid growth in prevalence in the next decade ( $77 \%$ to $146 \%$ ) will fall on low- and middle-income countries which have the least capacity to cope (World Health Organisation 2015). As population ageing continues to accelerate, the number of people with dementia is expected to nearly double every 20 years, growing to 65.7 million worldwide by 2030 (Alzheimer's
Association 2013). Despite wide variations in the proportions of formal and informal care arrangements for people with dementia in different regions of the world, there are similar social and economic costs (World Health Organisation 2014).

Dementia is classified in the fifth edition of the Diagnostic and Statistical Manual of Mental Disorders (DSM-5) (American Psychiatric Association 2013) as a major neurocognitive disorder, encompassing a range of degenerative conditions characterised by progressive decline in cognition and impairment in function. It is typically irreversible; currently there is no approved treatment available to prevent progression or to cure the disease. The most common type of dementia is Alzheimer's disease (approximately $50 \%$ to $75 \%$ ), which occurs usually after 65 years of age and is characterised by the accumulation of abnormal proteins in the brain in the form of amyloid plaques and neuritic tangles (Alzheimer's 
Disease International 2013). The next most common type is vascular dementia (20\% to $40 \%$ ), which results from decreased blood flow and hence impaired supply of oxygen and nutrients to the brain. Brain damage from major vascular events usually progresses in a step-wise fashion of deterioration and stabilisation, but disease of the small intracerebral vessels typically presents as dementia with a more insidious course. Death often occurs following a major stroke (Alzheimer's Association 2014a). Dementia with Lewy bodies (DLB) and dementia in Parkinson's disease (PDD) are closely related conditions in which degeneration of nerve cells is again thought to be due to abnormal protein deposits, in this case in the form of intracellular Lewy bodies (Alzheimer's Australia 2013). Frontotemporal dementia (FTD) often commences between 40 and 65 years of age, occurs in $5 \%$ to $10 \%$ of cases of dementia, and is associated with a variety of pathologies (Alzheimer's Australia 2013). With older age, the cause of dementia is more likely to be mixed, with combinations of Alzheimer's and vascular pathology being most common, although other mixed pathologies also occur. Early-onset dementia describes any form of dementia occurring in people under 65 years of age, with the main subtypes being alcohol-related dementia (22\%), Alzheimer's disease $(16 \%)$, frontotemporal dementia (13\%) and vascular dementia (10\%) (Draper 2011). Other forms of dementia occur with Korsakoff Syndrome, Huntington's disease, traumatic brain injury and HIV/AIDS (Alzheimer's Association 2014b).

There are often subtle changes in a person's behaviour and function before the formal diagnostic criteria for dementia are met. Symptoms typically include impairment of language, memory, perception, personality and cognitive skills. As the disease progresses there will be a decline in intellect, judgement, social skills, ability to manage daily functions and socially-tolerated emotional reactions. Some of the psychological responses in people with dementia include depression, psychosis, aggression and wandering from home (Alzheimer's Association 2013). Criteria for diagnosing dementia are outlined in the DSM-5 (American Psychiatric Association 2013). Cognitive changes must be assessed relative to the person's background cognitive ability, using culturally-sensitive and validated measures of physical and psychosocial function. This is especially important for people outside societal 'norms', including high-functioning individuals (who may not fit usual diagnostic criteria for dementia), those with special needs such as people with cultures and languages different from the mainstream society, and those with younger onset dementia and intellectual disability. Cognitive assessments need to be supplemented by a detailed longitudinal account, provided by an informant, of the person's health and social history with a focus on functional impairment. When appropriate measurement and interviewing styles are utilised in a quiet, safe and relaxed environment, people with dementia can provide reliable self-reports about their daily living activities and past life experiences. Utilising reminiscence during the interview assists in attending to cues and explicit references to symptoms conveyed by the person (Clare 2005; Clark 2008;
Kolanowski 2007). A physical examination and clinical investigations should also be conducted to rule out reversible causes.

\section{Description of the intervention}

Person-centred approaches to dementia care are based on the notion that the unique identity, personality and subjective reality of person living with dementia remain, despite the presence of a disease process (Kitwood 2007). Carl Rogers, one of the 20th century's most influential humanistic psychologists, founded the person-centred approach to care. "Rogers proposed that a person-centred approach, based on acceptance, caring, empathy, sensitivity, and active listening, promotes optimal human growth" (Brownie 2013 , p.2). As a response to the increasing disenfranchisement of people living with dementia in long-term care homes, social psychologist Tom Kitwood (Kitwood 2007) developed a model of person-centred care. His model challenged the notion that dementia leads to a loss of the person and, thus, to faulty interpretations of symptoms associated with dementia. Person-centred care operates from a perspective that many of these symptoms, such as agitation and aggression, are a result of the person's interactions within the psychosocial world. The 'social-psychological theory of personhood in dementia' (Kitwood 2007) is the basis for the person-centred care model, proposing that people exist in a social, relational context, and that positive and enriching interpersonal relationships can prevent the disabling effects of dementia and promote a sense of well-being (Brooker 2003; Davis 2004; Dewing 2008). The theory recognises the persistence of 'personhood' despite other losses that are occurring in dementia; supported by evidence that people with dementia retain a sense of identity (Rankin 2005) and the ability to communicate their wants, needs and desires (Mayhew 2001). Person-centred care, therefore, aims to support the person's identity by meeting their needs for love, attachment, comfort, occupation and inclusion. When these psychosocial needs are met, the person will have a sense of self-worth and feel valued, and there will be fewer expressions of ill-being, which are generally expressed in various forms of agitated and/or apathetic behaviour (Brooker 2007a; Slater 2006).

Person-centred care focuses its attention on maintaining and nurturing personhood. Person-centred care achieves its goal by adherence to a set of guiding principles:

1. Create and strengthen a positive relationship with the person through warm and accepting human contact.

2. Communicate respectfully, value and honour the person.

3. Treat the person as a sentient and unique human being, by valuing their innate nature and assisting them to retain their remaining strengths.

4. See the person's world from their perspective in all interactions with them.

5. Help the person to feel socially confident and to maintain emotional attachment to others. 
6. Assist the person to have choice and to make decisions about their own life.

7. Make use of the person's positive memories to help improve their self-esteem and maintain their identity.

8. Treat the person's behaviour as a way of communicating their feelings and needs and respond to the behaviour with empathy (Brooker 2007a).

Implementing person-centred care means promoting a system of social relationships that have the potential to mitigate the disabling effects of dementia through adherence to these principles in all interactions with the person (Davis 2004). The model relies on carers 'knowing the person' with dementia, understanding their unique life story, values and belief systems, preferences and idiosyncratic needs. This personal knowledge enables carers to engage with the person with dementia and form meaningful relationships with them. As a result the person with dementia is more secure in their environment, feels acknowledged as a person and is, therefore, less likely to become distressed or agitated.

Achieving these desirable outcomes is not only a matter of individuals learning the skills of person-centred care. It requires organisational leadership at all levels in order to address the structural barriers that prevent the adoption and maintenance of system-wide person-centred service, whether the system is a hospital ward, a day centre or a residential care home. The model is best implemented when the entire system is framed by policies to guide implementation across the organisation. All systems must operate to support personhood as the primary aim. This change in ethos requires organisational commitment to person-centred care and the education, training and supervision of all people involved in supporting the person's activities of daily living (Alzheimer's Disease International 2014). A shift in the system's loci of authority, control and power may, therefore, be required to support a person-centred service.

A number of different aspects of the organisation have been targeted in implementing person-centred care, including: organisational culture (Caspar 2009; Kane 2007); management systems (Miller 2010; Tellis-Nayak 2007); quality monitoring and improvement systems (Brooker 2007a); staff education and training (Chenoweth 2009; Fossey 2006; Stein-Parbury 2012); care planning prioritisation (Ashburner 2004); practice guidelines for specific issues such as resistance during personal care (Cohen-Mansfield 1999); care monitoring and staff support models such as Dementia Care Mapping (Brooker 2005; Fulton 2006); enriched activity programs (Brooker 2007b); and nursing guidelines (McCormack 2006).

\section{How the intervention might work}

As described above, person-centred care challenges the conventional constructions of dementia that can result in depersonalisation, treating the person as if they are no longer 'there' and focusing on their deficits (Davis 2004). A person-centred approach en- ables carers to interpret the behaviours of a person with dementia, not as a function of their illness, but rather as a response to what is happening to and occurring around them in their psychosocial milieu. Carers come to understand that it is how the person with dementia is being regarded and treated that can trigger behaviours, like aggression and resistance, during care events.

The review will consider person-centred interventions within the organisation which aim to support one or more of the 'VIPS' constructs (Røsvik 2013):

- Valuing: asserting the absolute value of the person's humanity.

- Individual care: recognising and responding to the person's uniqueness.

- Perspective: interpreting the person's actions/behaviour from their perspective.

- Social psychology: prioritising the person's well-being.

The VIPS constructs are relevant because they operate to support person-centred care across the entire organisation, by enabling the organisational structures that promote implementation.

\section{Why it is important to do this review}

Internaionally, people with dementia will eventually need some level of formal health and social care assistance, to complement and/or replace the care and support provided by their families (Alzheimer's Disease International 2014). Formal health and social care services operate in complex systems, which are bounded by the political, policy, economic, social and cultural frameworks unique to each culture and geographical region. While there is no unitary system and level of support that is suitable for all people with dementia, it is incumbent on governments and societies to ensure that, as far as possible, health and social care services are available to people with dementia and their families, and that services are dementia- inclusive (World Health Organisation 2014).

Family care for people with dementia is still common practice in most middle- and lower-income countries. In middle- and higherincome countries, the range of services available for the person and their family can include family support and respite, home help, personal care, palliative care, residential and assisted living support, long-term care assistance and nursing care, and hospital care. Each type of service has its own philosophical and policy framework, financial arrangement and requirements in service provision (Alzheimer's Association 2013). Despite these differences, formal service providers have a mandate to provide quality, evidencebased care and support to people with dementia and their families (American Psychiatric Association 2013). When services are dementia-inclusive, that is, they focus effort on adapting services to the needs of the individual, they can improve life for the person and their carer/s (Brownie 2013; León-Salas 2013; NICE 2010). A small number of dementia care service models have proved effective (Beerens 2013). 
One care model which has international appeal and evidence for its effectiveness and efficacy is the person-centred approach advocated by Tom Kitwood (Kitwood 1997). While there have been some criticisms of the model within the context of care relationships (Adams 2005; Nolan 2006b; Price 2006), the level of interest in person-centred care is growing. It has been adapted for use in acute, community and long-term care contexts in different cultures and societies (Aged Care Branch 2011; Brownie 2013; Wylie 2002). Person-centred care has been used as a general approach to improve specific aspects of care and treatment for people with dementia (Brooker 2007a; Fossey 2006; Sloane 2004), to improve their well-being (Chenoweth 2009; van de Ven 2013) and in helping to reduce various forms of agitation (Cohen-Mansfield 2012). Different approaches/techniques to implementing person-centred care have also proved helpful (Beavis 2002; Brownie 2013).

In many countries, including Australia, the United States, parts of South America and the Caribbean, Canada, the United Kingdom (UK), the Netherlands, Hong Kong, South Korea, Japan and across Scandinavia, quality dementia care is a national health and social priority. The governments of these nations are actively promoting person-centred dementia and aged care (Alzheimer's Association 2013). Given the investments that governments and service providers are making in developing dementia-inclusive services and communities, it is timely to undertake a rigorous review of the different person-centred models and care approaches that are operating across the world. It will be important to advise governments, the health and aged care industries and consumers on the outcomes of these different person-centred models and care approaches for people with dementia, their families and service providers.

\section{O B J E C T I VES}

Primary objective: To determine the effectiveness of organisationwide interventions to implement person-centred care for people with dementia, in relation to reduction of behavioural symptoms such as agitation and depression, improvement in quality of life and functional capabilities, alterations in the use of restraint (physical and/or chemical) and reduction in adverse events.

Secondary objective: To identify the variety, quality, and feasibility of person-centred care approaches for people with dementia, with specific reference to organisational factors which promote and constrain the implementation of person-centred care.

\section{MET HODS}

\section{Criteria for considering studies for this review}

\section{Types of studies}

We will include all published and unpublished randomised controlled trials (RCTs), including cluster-randomised trials (CRTs), recognising that this limits the review by excluding findings from other designs using qualitative methods. Trials will be included even if the method of sequence generation is sub-optimal ('quasirandomised') or unclear, however the inadequacies of the allocation method will be considered a source of selection bias.

\section{Types of participants}

Eligible participants will have been diagnosed with dementia according to the appropriate editions of the Diagnostic and Statistical Manual of Mental Disorders (DSM) (American Psychiatric Association 2013) and the International Classification of Diseases (ICD) (World Health Organisation 2015). If diagnostic information on participants is not described in potentially-relevant studies, study authors will be asked for additional diagnostic information. Participants will be receiving formal dementia care from an organisation which may be an individual care home, a chain of care homes, a hospital or a care agency providing formal care in these organisations.

\section{Types of interventions}

We will include interventions which are explicitly intended to promote the implementation of person-centred care at the organisational level, according to the VIPS constructs (Røsvik 2013; see How the intervention might work). Interventions may target one or more of: organisational culture; management systems; quality monitoring and improvement systems; staff education and training; and care planning prioritisation. They may also include nursing guidelines or practice guidelines for specific issues such as resistance during personal care, or specific techniques which aim to facilitate person-centred care, including care monitoring and staff support models such as Dementia Care Mapping (Brooker 2011). We will not include interventions with an individual focus, such as personalised activity programmes. The control interventions may either be 'care as usual' or 'standard care'. Studies that compare different approaches to implementing person-centred care will also be considered.

\section{Types of outcome measures}

\section{Primary outcomes}

- Frequency and severity of behavioural symptoms measured with either multi-faceted scales such as the Neuropsychiatric Inventory (Cummings 1994), scales specific to agitation such as the Cohen-Mansfield Agitation Inventory (Cohen-Mansfield 1999), or mood scales such as the Cornell Scale of Depression in Dementia (Alexopoulos 1988). 
- Quality of life, as measured by validated dementia quality of life instruments.

\author{
Secondary outcomes \\ - Function in activities of daily living. \\ - Use of physical/mechanical restraint. \\ - Use of chemical restraint including psychotropics, \\ antihistamines, and sedatives. \\ - Adverse events such as delirium, falls and related injuries, \\ and pressure ulcers.
}

\section{Search methods for identification of studies}

Relevant studies will be identified by searching various databases.

\section{Electronic searches}

We will search ALOIS (www.medicine.ox.ac.uk/alois) - the Cochrane Dementia and Cognitive Improvement Group's (CDCIG) specialised register.

ALOIS is maintained by the Trials Search Co-ordinator for the CDCIG, and contains studies that fall within the areas of dementia prevention, dementia treatment and management, and cognitive enhancement in healthy elderly populations. The studies are identified through:

1. Monthly searches of a number of major healthcare databases: MEDLINE, EMBASE, CINAHL, PsycINFO and Lilacs;

2. Monthly searches of a number of trial registers: ISRCTN; UMIN (Japan's Trial Register); the WHO portal (which covers ClinicalTrials.gov; ISRCTN; the Chinese Clinical Trials Register; the German Clinical Trials Register; the Iranian Registry of Clinical Trials and the Netherlands National Trials Register, plus others);

3. Quarterly search of The Cochrane Library's Central Register of Controlled Trials (CENTRAL);

4. Six-monthly searches of a number of grey literature sources: ISI Web of Knowledge Conference Proceedings; Index to Theses; Australasian Digital Theses.

To view a list of all sources searched for ALOIS see About ALOIS on the ALOIS website (www.medicine.ox.ac.uk/alois).

Details of the search strategies run in healthcare bibliographic databases, used for the retrieval of reports of dementia, cognitive improvement and cognitive enhancement trials, can be viewed in the 'Methods used in reviews' section within the editorial information about the Cochrane Dementia and Cognitive Improvement Group on The Cochrane Library.

We will run additional searches in MEDLINE, EMBASE, PsycINFO, CINAHL, ClinicalTrials.gov and the WHO Portal/ ICTRP to ensure that the searches for each suite of reviews is as comprehensive and as up-to-date as possible. The search strategy that will be used for the retrieval of reports of trials from MEDLINE (via the Ovid SP platform) can be seen in Appendix 1.

\section{Searching other resources}

We will also:

- handsearch reference lists of all included studies, relevant systematic reviews and other relevant publications;

- contact authors of relevant studies or reviews to clarify reported published information and seek unpublished data;

- conduct cited reference searches for all included studies, in ISI Web of Knowledge.

\section{Data collection and analysis}

The first review author (LC) will execute the search strategy as described, supported by the second and third authors (JS-P, SL). Any studies on PCC undertaken by the review authors that are deemed eligible for inclusion in the review will be assessed for study selection, extract data and 'Risk of bias' by an independent reviewer Professor Elizabeth Beattie (EB).

\section{Selection of studies}

The search results will be merged using reference management software and duplicate records removed. Two review authors (LC and JS-P) will independently screen the search results at three levels: (1) titles, (2) abstracts to assess which studies satisfy the inclusion criteria, and (3) full text copies of the papers that are potentially relevant. Obviously irrelevant reports will be removed. Multiple reports of the same study will be linked. If the paper cannot be assessed for relevance based on title or abstract, the full text will be obtained. When suitability of a study is unclear, after examining the full text the corresponding author will be contacted to request clarification or additional information, or both.

\section{Data extraction and management}

Two review authors (LC and JS-P) will extract data independently using the Template for Intervention Description and Replication (TIDieR) checklist (Hoffmann 2014). Any discrepancies will be resolved by discussion. The third and fourth review authors (SL and WYA) will be consulted in the case of persistent disagreement.

\section{Assessment of risk of bias in included studies}

Two review authors (LC and JS-P) working independently will assess the methodological quality of the included studies using the Cochrane 'Risk of bias' assessment tool (Higgins 2011). We will assess studies for random sequence generation, allocation concealment, blinding of participants and personnel, blinding of outcome assessment, incomplete outcome data, selective reporting 
and anything else that might lead to a systematic underestimate, or overestimate, of the difference between groups. Two review authors (SL and WYA) will assess the risk of bias independently and discrepancies will be resolved by discussion. An arbitrator will be consulted in the case of persistent disagreement.

\section{Measures of treatment effect}

All results will be presented with $95 \%$ confidence intervals. Dichotomous data: The effect measure for dichotomous outcomes will be the odds ratio.

Continuous data: The effect measure for continuous data will be the mean difference where arithmetic means and standard deviations are provided. If the same outcome is measured using different scales, then the standardised mean difference will be used. Geometric mean ratios will be used if continuous outcomes are summarised using geometric means. Medians and ranges will be reported in a table.

Count data: Effect measures for counts and rates will be rate ratios. Time-to-event data: Time-to-event data will be compared using hazard ratios.

\section{Unit of analysis issues}

We anticipate including cluster randomised trials. Where these report analyses which account for cluster randomisation, we will check that the method used was appropriate. If no correct analysis accounting for the cluster randomisation is reported, then we will attempt to re-analyse the data following the Cochrane Handbook guidelines (Higgins 2011). Where studies report outcomes at multiple time points, we will consider meta-analysis using methods described by Peters and Mengersen (Peters 2008), depending on the availability of summary effects, number of repeated outcome measures, between-subjects and within-subjects variance, difference between outcome measures at different time-points and slope estimate for trend. Meta-analyses will be conducted in the following circumstances:

- given time point, where most studies report one given time point and others report multiple time points;

- first/last time point, where most studies report two time points and a small number of studies report three or more time points; and

- all time points, where most studies report three or more time points.

If a study does not report appropriate results, we will not re-analyse the data because no estimate of within-subjects variation can be obtained and any re-analysis would underestimate, or overestimate, the standard error of the effect sizes. Therefore, as suggested in the Cochrane Handbook guidelines, we will present the results reported in the original papers only.

\section{Dealing with missing data}

Authors will be contacted and asked to supply the study protocol and full information for any outcomes reported inadequately. In addition, for outcomes mentioned in articles or protocols but not reported, we will ask authors to clarify whether those outcome measures were in fact analysed, and if so to supply the data. The completeness of outcome data for each main outcome will be described including attrition and exclusions from the analysis. We will report parameters such as the numbers in each intervention group (compared with total randomised participants), reasons for attrition/exclusions and any re-inclusions.

\section{Assessment of heterogeneity}

We will report variability between the included studies in terms of study design, participants, settings, interventions, comparisons, outcome assessment and outcome measures.

Substantial statistical heterogeneity will be suggested by the following indicators: poor overlap of confidence intervals on forest plots; high $\mathrm{Chi}^{2}$ values, $\mathrm{P}>0.10$, and $\mathrm{I}^{2}$ statistic $>50$.

\section{Assessment of reporting biases}

The possibility of selective outcome reporting will be examined and reported.

\section{Data synthesis}

We will appraise the variability across studies (in terms of participants, interventions, comparisons and outcomes) in order to make rational decisions about pooling data, or making particular comparisons across included studies. This assessment will include clinical heterogeneity and methodological heterogeneity. If studies are found to be sufficiently similar to ensure a clinically meaningful answer, we will use RevMan software to pool the results. If substantial heterogeneity is found, we will report results in a narrative form. Where applicable, we will calculate the relative risk and mean differences with $95 \%$ confidence intervals for categorical and continuous data, respectively. We will use a randomeffects model to take into account the (expected) heterogeneity of the various studies. We will examine data from cluster trials with unit of analysis errors according to the Effective Practice and Organisation of Care (EPOC 2015) guidelines, and absolute risk differences will be used.

\section{Subgroup analysis and investigation of heterogeneity}

Subgroup analysis, where possible, will be conducted on participant populations, in order to make comparisons between subgroups based on factors such as participant features, or subsets of studies (e.g. study setting and its geographical location). 


\section{Sensitivity analysis}

If sufficient trials that satisfy the inclusion criteria are identified, we will conduct sensitivity analyses regarding risk of bias to investigate the robustness of the results in relation to the quality components. The effect of missing data will also be investigated using sensitivity analyses.

\section{Presentation of results - 'Summary of findings' table}

For each outcome, we will assess the overall quality of the evidence using the GRADE (Grades of Recommendation, Assessment, Development and Evaluation) approach. The GRADE approach defines the quality of a body of evidence as the extent to which one can be confident that an estimate of effect or association is close to the true quantity of specific interest (Schünemann 2011a). We will present the main results of the review in 'Summary of findings' tables, which provide key information concerning the best estimate of effect of the interventions examined, and the quantity and the quality of the evidence behind each estimate (Schünemann 2011b). We plan to include the following main outcomes in the 'Summary of findings' tables:

1. Frequency and severity of agitation.

2. Multi-faceted behaviours.

3. Mood.

4. Quality of life.

5. Function in activities of daily living.

6. Use of restraint, physical and/or chemical.

7. Adverse events.

\section{R E F E R E N C E S}

\section{Additional references}

\section{Adams 2005}

Adams T. Living with dementia 1984-2004: from personcentred care to relationship centred care. Generations Review 2005; 15:4-7.

\section{Aged Care Branch 2011}

Aged Care Branch, Victorian Department of Health. Dementia-friendly environments: a guide for residential care. Canberra, Australia: Australian Government Department of Health, 2011.

\section{Alexopoulos 1988}

Alexopoulos GS, Abrams RC, Young RC, Shamoian CA. Cornell scale for depression in dementia. Biological Psychiatry 1988;23:271-84.

Alzheimer's Association 2013 Alzheimer's Association. Alzheimer's Facts and Figures 2013. Chicago, USA: Alzheimer's Association, 2013.

Alzheimer's Association 2014a Alzheimer's Association. Vascular Dementia. http:// www.alz.org/dementia/vascular-dementia-symptoms.asp 2014.

Alzheimer's Association 2014b

Alzheimer's Association. The search for Alzheimer's causes and risk factors. http://www.alz.org/research/science/ alxheimers_disease_causes.asp 2014.

Alzheimer's Australia 2013 Alzheimer's Australia. Collaborating for a better future for Australians living with Alzheimer's disease. Outcomes from a multidisciplinary roundtable discussion. Tasmania, Australia: Alzheimer's Australia, 2013.

Alzheimer's Disease International 2013

Alzheimer's Disease International. World Alzheimer Report 2013. Journey of Caring. An analysis of long-term care for dementia. London, UK: Alzheimer's Disease International, 2013.
Alzheimer's Disease International 2014

Alzheimer's Disease International. Dementia in the Asia Pacific Region. London, UK.: Alzheimer's Disease International, 2014.

American Psychiatric Association 2013 American Psychiatric Association. Diagnostic and Statistical Manual (DSM-5). 5th Edition. Washington DC: American Psychiatric Association, 2013.

Ashburner 2004

Ashburner C, Meyer J, Johnson B, Smith C. Using action research to address loss of personhood in a continuing care setting. Illness, Crisis, \& Loss 2004;12:23-37.

Beavis 2002

Beavis D, Simpson S, Graham I. A literature review of dementia care mapping: Methodological considerations and efficacy. Journal of Psychiatric and Mental Health Nursing 2002;9:725-36.

Beerens 2013

Beerens HC, Zwakhalen SMG, Verbeek H, Ruwaard D, Hamers JPH. Factors associated with quality of life of people with dementia in long-term care facilities: a systematic review. International Journal of Nursing Studies 2013;50:1259-70.

Brooker 2003

Brooker D. What is person-centred care in dementia?. Reviews in Clinical Gerontology 2003;13:215-22.

Brooker 2005

Brooker D. Dementia care mapping: a review of the research literature. The Gerontologist 2005;45:11-8.

Brooker 2007a

Brooker D. Person-centred Dementia Care: Making Services Better. Jessica Kingsley Publishers, 2007.

Brooker 2007b

Brooker DJ, Woolley RJ, Lee D. Enriching opportunities for people living with dementia in nursing homes: an 
evaluation of a multi-level activity-based model of care. Aging and Mental Health 2007;11:361-70.

\section{Brooker 2011}

Brooker D. Dementia care mapping. In: Abou-Saleh MT, Katona C, Kumar A editor(s). Principles and Practice of Geriatric Psychiatry. 3rd Edition. Wiley, 2011:157-61.

\section{Brownie 2013}

Brownie $S$, Nancarrow $S$. Effects of person-centered care on residents and staff in aged-care facilities: a systematic review. Clinical Interventions in Aging 2013;8:1.

\section{Caspar 2009}

Caspar S, O'Rourke N, Gutman GM. The differential influence of culture change models on long-term care staff empowerment and provision of individualized care. Canadian Journal on Aging/La Revue canadienne du vieillissement 2009;28:165-75.

\section{Chenoweth 2009}

Chenoweth L, King MT, Jeon Y-H, Brodaty H, SteinParbury J, Norman R, et al. Caring for Aged Dementia Care Resident Study (CADRES) of person-centred care, dementia-care mapping, and usual care in dementia: a cluster-randomised trial. The Lancet Neurology 2009;8: $317-25$.

Clare 2005

Clare L, Woods B. Awareness in dementia: a subject for scrutiny. Aging \& Mental Health 2005;9:384-5.

\section{Clark 2008}

Clark PA, Tucke SS, Whitlatch CJ. Consistency of information from persons with dementia: an analysis of differences by question type. Dementia 2008;7:341-58.

\section{Cohen-Mansfield 1999}

Cohen-Mansfield J. Measurement of inappropriate behavior associated with dementia. Journal of Gerontological Nursing 1999;25:42-51.

\section{Cohen-Mansfield 2012}

Cohen-Mansfield J, Thein K, Marx MS, Dakheel-Ali M, Freedman L. Efficacy of nonpharmacologic interventions for agitation in advanced dementia: a randomized, placebocontrolled trial. The Journal of Clinical Psychiatry 2012;73: 1255-61.

\section{Cummings 1994}

Cummings JL, Mega M, Gray K, Rosenberg-Thompson S, Carusi DA, Gornbein J. The Neuropsychiatric Inventory: comprehensive assessment of psychopathology in dementia. Neurology 1994;44:2308-14.

\section{Davis 2004}

Davis DHJ. Dementia: sociological and philosophical constructions. Social Science \& Medicine 2004;58:369-78.

\section{Dewing 2008}

Dewing J. Personhood and dementia: revisiting Tom Kitwood's ideas. International Journal of Older People Nursing 2008;3:3-13.

\section{Draper 2011}

Draper B. Understanding Alzheimer's and Other Dementias. Woollahra: Longueville Books, 2011.

\section{EPOC 2015}

Effective Practice, Organisation of Care (EPOC). EPOC Resources for review authors. Oslo: Norwegian Knowledge Centre for the Health Services. http://epoc.cochrane.org/ epoc-specific- 2015.

\section{Fossey 2006}

Fossey J, Ballard C, Juszczak E, James I, Alder N, Jacoby R, et al. Effect of enhanced psychosocial care on antipsychotic use in nursing home residents with severe dementia: cluster randomised trial. BMJ 2006;332:756-61.

\section{Fulton 2006}

Fulton BR, Edelman P, Kuhn D. Streamlined models of dementia care mapping. Aging \& Mental Health 2006;10: 343-51.

\section{Higgins 2011}

Higgins JPT, Green S (editors). Cochrane Handbook for Systematic Reviews of Interventions Version 5.1.0 [updated March 2011].. The Cochrane Collaboration, 2011. Available from www.cochrane-handbook.org.

\section{Hoffmann 2014}

Hoffmann TC, Glasziou PP, Boutron I, Milne R, Perera R, Moher $\mathrm{D}$, et al. Better reporting of interventions: template for intervention description and replication (TIDieR) checklist and guide. BMJ 2014;348:g1687. [DOI: http:// dx.doi.org/10.1136/bmj.g1687]

Kane 2007

Kane RA, Lum TY, Cutler LJ, Degenholtz HB, Yu TC. Resident outcomes in small-house nursing homes: a longitudinal evaluation of the initial Green House Program. Journal of the American Geriatrics Society 2007;55:832-9.

\section{Kitwood 1997}

Kitwood T. The experience of dementia. Aging \& Mental Health 1997;1:13-22.

\section{Kitwood 2007}

Kitwood TM. Dementia reconsidered: the person comes first. Buckingham: Open University Press, 2007.

\section{Kolanowski 2007}

Kolanowski A, Hoffman L, Hofer SM. Concordance of selfreport and informant assessment of emotional well-being in nursing home residents with dementia. The Journals of Gerontology Series B: Psychological Sciences and Social Sciences 2007;62:P20-7.

\section{León-Salas 2013}

León-Salas B, Olazarán J, Cruz-Orduña I, Agüera-Ortiz L, Dobato José L, Valentí-Soler M, et al. Quality of life (QoL) in community-dwelling and institutionalized Alzheimer's disease (AD) patients. Archives of Gerontology and Geriatrics 2013;57:257-62.

Mayhew 2001

Mayhew PA, Acton GJ, Yauk S, Hopkins BA. Communication from individuals with advanced DAT: can it provide clues to their sense of self-awareness and wellbeing?. Geriatric Nursing 2001;22:106-10. 


\section{McCormack 2006}

McCormack B, McCance TV. Development of a framework for person-centred nursing. Journal of Advanced Nursing 2006;56:472-9.

Miller 2010

Miller SC, Miller EA, Jung H-Y, Sterns S, Clark M, Mor V. Nursing home organizational change: The "culture change" movement as viewed by long-term care specialists. Medical Care Research and Review 2010;67(4 Suppl):65S-81S.

\section{NICE 2010}

National Institute for Health and Care Excellence. Dementia: support in health and social care (Dementia quality standard 1). http://www.nice.org.uk/guidance/qs1 National Institute for Health and Care Excellence, 2010.

Nolan 2006b

Nolan M, Davies S, Brown J. Transitions in care homes: towards relationship-centred care using the 'Senses Framework'. Quality in Ageing and Older Adults 2006;7: $5-14$.

\section{Peters 2008}

Peters JL, Mengersen KL. Meta-analysis of repeated measures study designs. Journal of Evaluation in Clinical Practice 2008;14(5):941-50.

Price 2006

Price B. Exploring person-centred care. Nursing Standard 2006;20:49-56.

Rankin 2005

Rankin KP, Baldwin E, Pace-Savitsky C, Kramer JH, Miller BL. Self awareness and personality change in dementia. Journal of Neurology, Neurosurgery \& Psychiatry 2005;76: 632-9.

\section{Røsvik 2013}

Røsvik J, Brooker D, Mjorud M, Kirkevold Ø. What is person-centred care in dementia? Clinical reviews into practice: the development of the VIPS practice model. Reviews in Clinical Gerontology 2013;23(2):155-63.

\section{Schünemann 2011a}

Schünemann HJ, Oxman AD, Higgins JPT, Vist GE, Glasziou P, Guyatt GH. Chapter 11: Presenting results and 'Summary of findings' tables 2011. In: Higgins JPT, Green S editor(s). Cochrane Handbook for Systematic Reviews of Interventions Version 5.1.0 (updated March 2011). The Cochrane Collaboration, 2011.

\section{Schünemann 2011b}

Schünemann HJ, Oxman AD, Higgins JPT, Vist GE, Glasziou P, Guyatt GH. Chapter 12: Interpreting results and drawing conclusions. In: Higgins JPT, Green S editor(s). Cochrane Handbook for Systematic Reviews of Interventions Version 5.1.0 (updated March 2011). The Cochrane Collaboration, 2011.

\section{Slater 2006}

Slater L. Person-centredness: a concept analysis. Contemporary Nurse 2006;23:135-44.

Sloane 2004

Sloane PD, Hoeffer B, Mitchell CM, McKenzie DA, Barrick AL, Rader J, et al. Effect of person-centered showering and the towel bath on bathing-associated aggression, agitation, and discomfort in nursing home residents with dementia: A randomized, controlled trial. Journal of the American Geriatrics Society 2004;52:1795-804.

\section{Stein-Parbury 2012}

Stein-Parbury J, Chenoweth L, Jeon Y-H, Brodaty H, Haas M, Norman R. Implementing person-centered care in residential dementia care. Clinical Gerontologist 2012;35: 404-24.

\section{Tellis-Nayak 2007}

Tellis-Nayak V. A person-centered workplace: the foundation for person-centered caregiving in long-term care. Journal of the American Medical Directors Association 2007;8:46-54.

\section{van de Ven 2013}

van de Ven G, Draskovic I, Adang EM, Donders R, Zuidema SU, Koopmans RT, et al. Effects of dementia-care mapping on residents and staff of care homes: a pragmatic cluster-randomised controlled trial. PloS one 2013;8: e67325.

World Health Organisation 2014 World Health Organisation. Global Health Observatory Data Repository. World Health Organisation, 2014.

\section{World Health Organisation 2015}

World Health Organisation (WHO). International Statistical Classification of Diseases and Related Health Problems. World Health Organization, 2015.

\section{Wylie 2002}

Wylie K, Walton JA, Madjar I. Dementia care mapping: a person-centred, evidence-based approach to improving the quality of care in residential care settings. Geriaction 2002; 20(2):5-9.

* Indicates the major publication for the study 


\section{A P P E N D I C ES}

\section{Appendix I. MEDLINE search strategy}

1. exp Dementia/

2. Delirium, Dementia, Amnestic, Cognitive Disorders/

3. dement*.mp.

4. alzheimer*.mp.

5. (lewy* adj2 bod*).mp.

6. (chronic adj2 cerebrovascular).mp.

7. ("organic brain disease" or "organic brain syndrome").mp.

8. (cerebr* adj 2 deteriorat*).mp.

9. (cerebral* ${ }^{*}$ adj2 insufficient $\left.{ }^{*}\right) \cdot \mathrm{mp}$.

10. or/1-9

11. activity.ti,ab.

12. activities.ti,ab.

13. psychosocial.ti,ab.

14. non-pharmacological.ti,ab.

15. individually-tailor*.ti,ab.

16. personally-tailor*.ti,ab.

17. (individual or individuals or individually-cent*).ti,ab.

18. (meaning* OR meaningful*).ti,ab.

19. involvement.ti,ab.

20. (engagement or engaging).ti,ab.

21. occupational*.ti,ab.

22. personhood.ti,ab.

23. person-centred.ti,ab.

24. identity.ti,ab.

25. Personhood/ or Patient-Centred Care/

26. or/11-24

27. 10 and 26

28. randomized controlled trial.pt.

29. controlled clinical trial.pt.

30. randomly.ab.

31. groups.ab.

32. placebo.ti,ab.

33. randomi?ed.ti,ab.

34. ("double-blind"” or "single-blind").ti,ab.

35. (RCT or CCT).ti,ab.

36. or $/ 28-35$

37.27 and 36

Organisational interventions for promoting person-centred care for people with dementia (Protocol) 


\section{CONTRIBUTIONS OFAUTHORS}

\section{Lynn Chenoweth}

Conceived the review's focus and methodology with authors Stein-Parbury and Lapkin, consulted with author Wang on the review's methodology and the statistical methods, undertook the preliminary literature review on PCC processes and applications, wrote the first draft of the review proposal, reviewed all subsequent manuscript drafts, and completed the final manuscript.

\section{Jane Sten-Parbury}

Assisted author Chenoweth with conceiving the review's focus and methodology, assisted with the preliminary literature review on PCC processes and applications, contributed to all manuscript drafts and contributed to the final manuscript .

\section{Samuel Lapkin}

Assisted author Chenoweth with conceiving the review's focus and methodology, consulted with author Wang to decide on the review's statistical methods, reviewed all drafts and contributed to the final manuscript.

\section{Alex Wang}

Contributed expert advice on the review procedures and statistical methods, reviewed manuscript drafts and contributed to the final manuscript.

\section{DECLARATIONSOF INTEREST}

None known

\section{SOURCES OF SUPPORT}

\section{Internal sources}

- No sources of support supplied

\section{External sources}

- National Institute for Health Research (NIHR), UK.

This protocol was supported by the National Institute for Health Research, via Cochrane Infrastructure funding to the Cochrane Dementia and Cognitive Improvement group. The views and opinions expressed herein are those of the authors and do not necessarily reflect those of the Systematic Reviews Programme, NIHR, National Health Service (NHS) or the Department of Health 